\title{
A Simulation-Based Analysis of the Loss Process of Broadcast Packets in WAVE Vehicular Networks
}

\author{
Salvador Gonzalez and Victor Ramos \\ Department of Electrical Engineering, Universidad Autónoma Metropolitana, Mexico City 09340, Mexico \\ Correspondence should be addressed to Victor Ramos; victor.ramos@ieee.org
}

Received 15 June 2018; Revised 7 September 2018; Accepted 1 October 2018; Published 16 October 2018

Guest Editor: MoonBae Song

Copyright (C) 2018 Salvador Gonzalez and Victor Ramos. This is an open access article distributed under the Creative Commons Attribution License, which permits unrestricted use, distribution, and reproduction in any medium, provided the original work is properly cited.

\begin{abstract}
The IEEE 802.11p standard operates with the WAVE (Wireless Access in Vehicular Environments) system in vehicular ad hoc networks (VANETs). The broadcast process is used to send messages for safety and non-safety applications. A previous work on broadcast packets over the control channel proposes an analytical model to study the loss process. Even if such work does not consider all of the phenomena affecting the operation of vehicular networks, we can obtain a very good approximation of the performance that VANETs may exhibit. Regardless of its importance, this subject has been barely studied. Moreover, there is in the literature only a couple of contributions on this subject, being both analytical models. Therefore, we present in this paper an analysis of the loss process of broadcast packets on the control channel of VANETs over different scenarios. First, we consider a typical twoway scenario and then we analyze a scenario with intersections, both for different vehicle densities. We conduct a campaign of extensive simulations with the NS-3 simulator to study the average loss rate of broadcast packets, and then we compare our results with an analytical model proposed by Campolo et al. We prove the relationship among the contention window, the packet size, and the number of vehicles with the loss rate, including losses caused by noise, collisions, hidden terminal, and channel switching. Thus, we analyze the loss process validating the results obtained by Campolo et al. We find that there are additional factors affecting the loss rate, which cannot be captured with the analytical model. One key finding in this work is that the loss rate due to channel switching differs between both approaches. Also, we find bounds on the use of the control channel, with the loss rate and the traffic load in the network as parameters.
\end{abstract}

\section{Introduction}

Research in vehicular communications has been exponentially growing during the last years. It is expected that vehicular ad hoc networks (VANETs) will offer a wide variety of services such as traffic information, prevention against accidents, and entertainment just to mention a few. During 2006, the Wireless Access for Vehicular Environments (WAVE) initiative greatly contributed to the research on VANETs. To provide services on VANETs, it is necessary to account with a trustworthy infrastructure as well as with protocols that offer a good quality of service (QoS).

There are two possible communications infrastructures in VANETs, one for communications between a vehicle and a device located at roadside (V2I, vehicle to infrastructure), and another one for communications between vehicles (V2V, vehicle to vehicle). According to these two infrastructure types, one can be able to account with reliable information all the way; besides, any vehicle may contribute with information about the road status or any other information it wants to share.

To carry out the communication between the infrastructures, the Intelligent Transport Systems Committee of the IEEE Vehicular Technology Society introduced the IEEE 802.11p amendment [1]. This standard aims to propose efficient mechanisms to control the operation of upper layers using multiple channels without the knowledge of the physical layer parameters. It also describes the multichannel operation for routing and switching under different scenarios. The IEEE 802.11p standard is required by intelligent transport systems; it covers the physical layer as well as the link layer from the WAVE protocol stack [2].

The IEEE 802.11p committee has published a series of physical layer and medium access control layer specifications 
to allow vehicular communications in the $5.9 \mathrm{GHz}$ band. The physical layer is a variation of the IEEE 802.11a amendment, which employs 64 OFDM subcarriers. Among these, 52 are used for transmission comprising 48 data subcarriers and 4 pilot subcarriers. The pilot signals are used to find shift noise in frequency and phase. The short and long training symbols located at the head of each data packet are used for signal detection, frequency offset estimation, time synchronization, and channel estimation. A guard time in each OFDM data symbol removes the interference between symbols, which occurs because of multipath propagation. To decrease the effects of channel fading, the information bits are encoded and interleaved. The IEEE 802.11p physical layer executes exactly the same procedure than IEEE 802.11a for signal processing and also has the same specifications, except for the following changes:

(i) As we mentioned above, IEEE 802.11p operates in the $5.9 \mathrm{GHz}$ frequency band; it has a bandwidth of 75 $\mathrm{MHz}$ split in 7 channels of $7 \mathrm{MHz}$ each, leaving 5 $\mathrm{MHz}$ as a guard band. At the middle is the control channel, where all the messages needing reliability are broadcast. Low priority communications are carried out in the remaining channels, which are service channels. Besides, two adjacent service channels may be employed as a single $20 \mathrm{MHz}$ channel.

(ii) To provide support for long-distance communications, the standard defines four classes of Effective Isotropic Radiated Power (EIRP). The highest value is $44.8 \mathrm{dBm}(30 \mathrm{~W})$, which is for emergency vehicles.

(iii) To improve the tolerance to multipath fading propagation, a $10 \mathrm{MHz}$ bandwidth is employed. With this, the remaining IEEE 802.11p parameters are doubled compared with IEEE 802.11a.

(iv) When needed, to synchronize channel switching and keep all the vehicles and infrastructures in the control channel, a satellite navigation system may provide a global time reference such as UTC (Coordinated Universal Time).

To prioritize access in IEEE 802.11p, EDCA (Enhanced Distributed Channel Access) is used, which is originally proposed for IEEE 802.11e. This function includes the listen before talk (LBT) mechanism as well as a random backoff. Access to the prioritized channel is provided by four different classes of traffic: background (AC BK), best-effort (AC BE), voice (AC VO), and video (AC VI).

The EDCA mechanism is based on CSMA/CA (CarrierSense Multiple Access with Collision Avoidance). With this technique, a station wishing to transmit must sense the medium before making any transmission to determine if it is idle or busy. Particularly, a wireless station with a data flow to transmit among one of the classes (AC) just mentioned senses the medium before transmitting a frame. If the medium is busy, the station delays its transmission to avoid collisions with other stations. The backoff value is computed randomly following a uniform distribution in the interval $[0, \mathrm{CW}[\mathrm{AC}]]$, where $\mathrm{CW}[\mathrm{AC}]$ is the contention window for the $\mathrm{AC}$ class. During the first transmission, the value of $\mathrm{CW}[\mathrm{AC}]$ is set to $\mathrm{CW}_{\min }[\mathrm{AC}]$ and it is doubled at each retransmission up to a maximum limit of $\mathrm{CW}_{\max }[\mathrm{AC}]$. The backoff counter is decremented while the medium is idle at the end of each time slot. If the channel is detected busy while decrementing the backoff, then the decrement is stopped, and it will be resumed when the channel becomes idle during a certain period of time according to the AC value. The frame is transmitted when the backoff timer reaches a value of zero.

In this paper, we focus on the loss process of broadcast packets in IEEE 802.11p/WAVE vehicular networks. Despite its importance, this subject has been very little studied. In [3], Campolo et al. extend an analytical model proposed by themselves to characterize broadcast packet losses in this type of systems. The baseline model characterizes broadcast transmissions on the control channel assuming channel switching under ideal conditions; it does not discriminate different types of losses. Thus, the extended model in [3] considers an error-prone channel and different types of losses, such as losses due to collisions, channel errors, or the end of the interval of the control channel. Such an analytical model is, of course, a very valuable contribution to understand the loss process of broadcast packets in that type of systems.

Over the last few years, VANETs have been studied through analytical models, by simulation, and exceptionally with experimental testbeds [4]. To date, there are no contributions in this research direction to analyze the loss process of broadcast by means of simulation. In fact, most of the research work around VANETs is carried out with the aid of simulators. This is easily explained by observing the huge effort needed to design and implement a testbed for experimental analysis. Therefore, in this work we conduct an extensive simulations campaign on different VANET scenarios to validate the analytical model in [3]. We find up to a certain point a great similarity with Campolo's results; however, we also find that the expected loss rate does not correspond between both approaches, i.e., Campolo's and ours. Particularly, losses due to channel switching behave differently. Moreover, we are able to analyze four types of losses: hidden terminal, collision, noise, and channel switching. Finally, we find bounds on the use of the control channel as a function of the loss rate and the traffic load in the network.

The remaining of this paper is structured as follows: in Section 2 we describe the operation of broadcast, the models proposed in the literature, and the characterization of broadcast losses on WAVE systems. In Section 3, we depict the simulation process, the modules we added, and the modifications we make for our simulations. In Section 4, we describe the scenarios and the parameters used to evaluate the loss process on WAVE systems; we also present in this section the results obtained in our simulations. Finally, Section 5 sketches our conclusions and future work.

\section{Related Work}

The control channel is designated to transmit critical security messages, beacons, and messages for connection establishment of service channels (WSA, WAVE Service Advertisement). A traffic load in normal conditions for the control 
channel includes the generation of beacon periodic messages, as well as WSAs, and only on emergency cases security messages are sent to alert from danger events on the road.

Beacons are essential to support vehicular applications that require precise and timely information. Also, they are crucial to provide services such as neighbor discovery, proactive routing algorithms, or intelligent security broadcast strategies. To meet the requirements of such applications, the typical beacon generation rate is $10 \mathrm{~Hz}$ [5]; i.e., once every WAVE synchronization interval. This implies a huge load on the channel when the number of vehicles is large. To avoid such a load, we may find in the literature work proposing to adapt the transmission power [6] or to manage the beacon generation rate [7]. All the packets coming from the application layer are sent to the MAC layer only during the control channel interval. Due to the nature of beacons and the messages for communication establishment of service channels, it is valid to assume that their life time is limited to the duration of the interval of the control channel. After this period, frames that were not transmitted are discarded and, therefore, there is no need to model the MAC queues for broadcast messages. In [8], the authors evaluate an Intelligent Transportation System (ITS) application where the beacon frequency and the transmit power of nodes are the main parameters. Moreover, vehicle density is a key traffic parameter. The main finding is that the application's performance can be improved by finely tuning the beacon frequency along with the transmit power as a function of the traffic density.

2.1. Broadcast Models for the Control Channel. To evaluate the performance of the system, the commonly parameters considered are packet delay, collision probability, the success probability when receiving packets, and throughput. To assess such performance measures, several models have been proposed in the literature. In [9], the authors use a Markov Chain to model the successful delivery of packets for emergency messages and beacons. To prioritize channel access, different values for the contention window are used. This model considers only losses due to collisions and assumes the network is operating in saturation conditions. In [10], the authors propose a function for the distributed channel to analyze the generation and transmission of beacons to determine the probability of collision-free transmissions.

We may find in the literature more examples like these, but only few consider channel switching, which obviously impacts directly the network performance. In [11], an analytical model is proposed to evaluate the performance of the mechanism for channel coordination under different traffic patterns and channel intervals; however, this work focuses on unicast message transmission and not on broadcast, which is our main focus. In [12], the authors propose an analytical model to evaluate throughput and collision probability in an IEEE 802.11p network, where nodes always have packets to send; the model estimates throughput saturation ignoring packet priority. In [3], Campolo et al. propose a model to characterize broadcast packet losses on the control channel, which is a modification of a model proposed by the same authors in [13], where channel switching is considered. The drawback of the model in [13] is that there is no distinction between the cases where there are packet losses; thus, the authors add such a case in [3]. Later in [14], the authors add the effect of priority on the different types of messages. Finally, in [15] the author proposes two analytical models to evaluate the performance of IEEE 802.11p and IEEE 1609.4 protocols by considering channel switching. The models use Markov chains to characterize safety messages and WSA messages to study broadcasting reliability of safety-related messages on the control channel.

As we mentioned above, our aim is to study broadcast messages on the control channel; nevertheless, the following work is worth to mention even if it is not focused on the control channel or broadcast messages since it studies the importance of the contention window, which is a parameter we also study in this work. In [16], Jang and Feng propose a method to dynamically adapt the contention window to improve the throughput in IEEE 802.11p on the service channels. Similarly, in [17] Chrysostomou et al. propose to adapt the contention window also to increase throughput, while at the same time improving quality of service (QoS) for different message priorities. Moreover, in [18] Wang studies the impact of the contention window on throughput; even if it is not clear on which channel the work is focused, the throughput analysis is very interesting for different sizes of the contention window. In [19], Garg et al. evaluate the performance of a data delivery mechanism for Vehicular Ad Hoc Networks and Cognitive Radio (CR) Vehicular networks; they find that CR networks outperform VANETs when multiple types of packets are transmitted in a vehicle-to-vehicle environment. In [20], Taherkani and Pierre propose a multiobjective algorithm for congestion control in VANETs; their proposal is evaluated under highway and urban scenarios to find that it outperforms schemes like CSMA/CA, D-FPAV (DistributedFair Power Adjustment for Vehicular Environments), and CABS (Context Awareness Beacon Scheduling). Finally, the authors in [21] evaluate the influence of packet error models on VANET simulations. Thus, they measure loss rate, endto-end delay, and the number of hops; they also consider different densities of nodes and different channel capacities. The main finding is that in the best case the basic error model may obtain reliable results.

\subsection{Characterization of Packet Losses of Broadcast Messages} on the Control Channel. As cited before, in [3] Campolo et al. propose a model to characterize broadcast losses on the control channel, such a model is based on the IEEE 802.11p standard. Moreover, the authors assume the following: (1) all the nodes are within a reciprocal communication range, i.e., there is no hidden terminal; (2) the one-hop neighborhood does not change significantly due to vehicle mobility in a 50 ms interval, which is the duration of the control channel. With these conditions, the authors characterize all the possible events that can occur on the control channel during the broadcast process. Such possible events are the following: (a) the duration of the control channel is not sufficient to send the packet (res); (b) the packet is successfully transmitted (suc); 


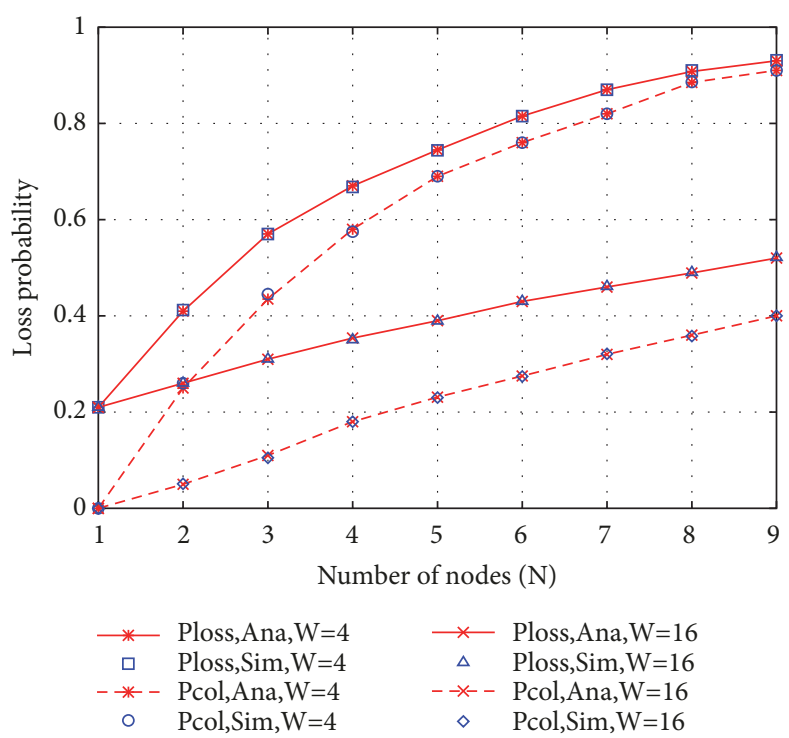

FIGURE 1: Probability of packet loss versus the number of nodes in the system with $W=4$ and $W=16$.

(c) the packet is lost because of noise (noise); and (d) collision during transmission ( $\mathrm{col}$ ).

To find the probability that $k$ out of $n$ vehicles transmit in slot $l$ of $w$, given that no attempt was made to transmit in the previous $l-1$ slots, Campolo et al. obtain (1). Such expression is modeled as a Bernoulli process, where $1 /(w-l+1)$ is the probability of uniformly choosing a slot from the $w-l+1$ available slots.

$$
\begin{aligned}
& P(l, n, w, k) \\
& =\left(1-\frac{l-1}{w}\right)^{n}\left(\begin{array}{l}
n \\
k
\end{array}\right)\left(\frac{1}{w-l+1}\right)^{k}\left(1-\frac{1}{w-l+1}\right)^{n-k}
\end{aligned}
$$

To model the average number of $e v$ events occurring in the system with $n$ vehicles, a contention window $w$, and $t$ remaining slots in the control channel interval, $X_{\mathrm{ev}}(t, w, n)$ is defined as follows:

$$
\begin{aligned}
& X_{e v}(t, w, n)=\sum_{l=1}^{\min (w, t)} P(l, n, w, 1)(1-B E R)^{L} \\
& \cdot\left[C_{s u c}+X_{e v}(t-l+1-s, w-l, n-1)\right] \\
& +P(l, n, w, 1)\left[1-(1-B E R)^{L}\right]\left[C_{n o i s e}\right. \\
& \left.+X_{e v}(t-l+1-c, w-l, n-1)\right]+\sum_{k=2}^{n} P(l, n, w, k) \\
& \quad \cdot\left[k C_{c o l}+X_{e v}(t-l+1-c, w-l, n-k)\right]
\end{aligned}
$$

The first two terms of (2) represent the probability that one out of $n$ vehicles choose a backoff value from the $w$ slots and it will successfully transmit in slot $l$ with probability $(1-\mathrm{BER})^{L}$, or it will experience one error in the channel with probability $1-(1-\mathrm{BER})^{L}$. The term $X_{\mathrm{ev}}(t-l+1-s, w-l, n-1)$ represents the average number of $e v$ events in the remaining $t-l+1-s$ slots. Moreover, for the second contribution of $X_{\mathrm{ev}}(t, w, n)$ it represents the average number of collisions when $k$ out of $n$ vehicles transmit in slot $l$, with $k>1$. $C_{i}$, with $i \in(s u c$, noise, $c o l$ ) is equal to 1 if $i=e v$; otherwise, it is equal to 0 . According to (1) and (2), the probability of any event $\mathrm{ev}$ is obtained as follows:

$$
P_{\mathrm{ev}}=\frac{X_{\mathrm{ev}}(t, w, n)}{n}
$$

Thus, (4) gives the probability of the res event as follows:

$$
P_{\text {res }}=1-P_{\text {suc }}-P_{\text {noise }}-P_{\text {col }} \text {. }
$$

Obviously, the total number of losses may be obtained as

$$
P_{\text {loss }}=1-P_{\text {suc }} \text {. }
$$

To validate their model, Campolo et al. compare it with simulations done in Matlab. The simulation parameters are as follows: $\mathrm{BER}=0.0001$; contention window, $w=4$ and $w=16$; packet size of 300 bytes; slot length of $16 \mu \mathrm{s}$; Short InterFrame Space (SIFS) of $32 \mu \mathrm{s}$; header length (time it takes for the physical layer to place its header to transmit) of $40 \mu \mathrm{s}$; and transmission rate of $3 \mathrm{Mb} / \mathrm{s}$. The results of the simulation versus the analytical model are shown in Figure 1.

As we see, the results show that the proposed analytical model is very close to the simulation results done in Matlab. Besides, we may see the performance of broadcast in IEEE 802.11p on the control channel. Clearly, as the number of nodes increases in the system, the collision probability increases as well. Moreover, we can observe also that as the contention window increases, the loss probability and the number of collisions decreases. Nevertheless, the contention window cannot overgrow since it is limited by the $50 \mathrm{~ms}$ allocated for the control channel. Thus, the authors show the system's behavior with larger values for the contention window and larger packet sizes as well. Figure 2 shows the corresponding behavior [3]. 


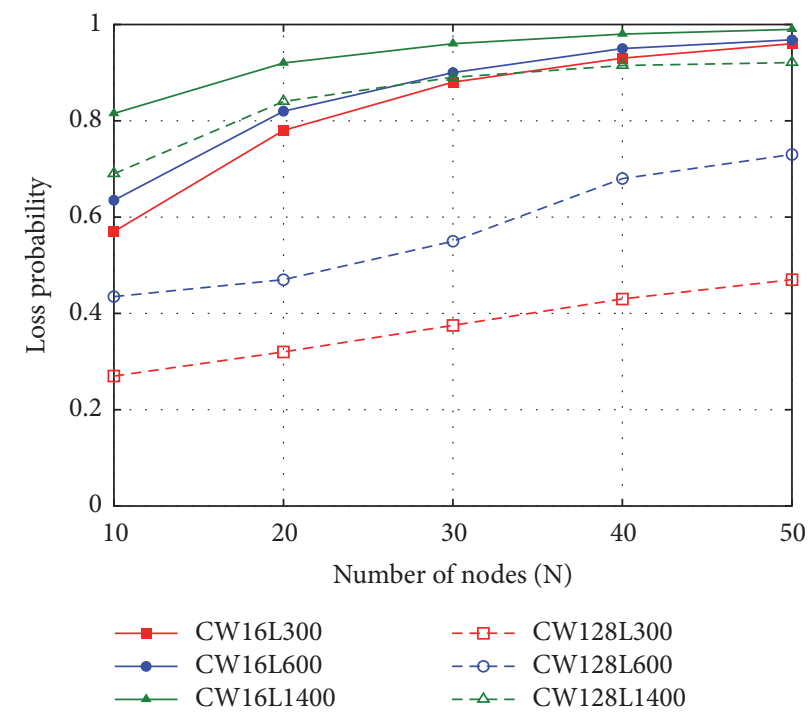

FIGURE 2: Probability of packet loss versus the number of nodes in the system with $W=16$ and $W=128$.

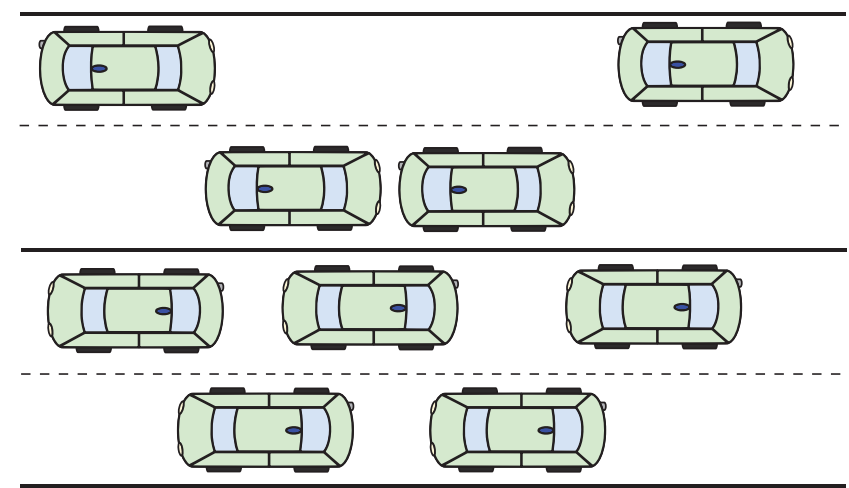

Figure 3: Simple two-way scenario.

\section{Evaluation}

To evaluate the performance of WAVE systems, adequate conditions must be chosen. In this work, we consider two vehicular scenarios for which we obtain the percentage of losses for each set of parameters. In this way, we obtain a clearer vision of WAVE systems. Hence, we explore the system under conditions going from a very low to a very high vehicular density.

3.1. Simple Two-Way Scenario. To analyze the system's behavior in scenarios like highways or freeways, we choose a simple two-way scenario, where we set two lanes in one direction and two lanes in the opposite sense. In this way, we have communications among vehicles in convoy and in opposite senses. This scenario is typical and is considered very representative in related work. Our scenario is $1 \mathrm{~km}$ long and has a separation between lanes with opposite senses of $10 \mathrm{~m}$. Figure 3 shows the simulated scenario.

3.2. Scenario with Crossing Lanes. It is also very important to consider scenarios that represent typical situations on cities.
Thus, we consider also a scenario with crossing lanes to model a larger number of vehicles within the transmission range. In this way, we can observe how the system's behavior changes compared to the simple two-way scenario. This case is particularly interesting to study since we can easily find it in real life. Surprisingly, this case has not been very studied in the related work. Therefore, our scenario has a length of 340 $\mathrm{m}$ from left to right as well as from to top to bottom. Figure 4 illustrates such a scenario.

3.3. Performance Measures. In this work, we focus on the percentage of packet loss in the system. This is because we work on the control channel and so, it is important that such packets arrive to the vehicles. Such an importance lies on the nature of the transmitted packets, such as packets for security applications, beacons, and messages to establish communication between service channels.

3.4. System Considerations. Regarding our simulations, we consider the transmission of just one packet in every interval of the control channel. All the vehicles transmit, but they 


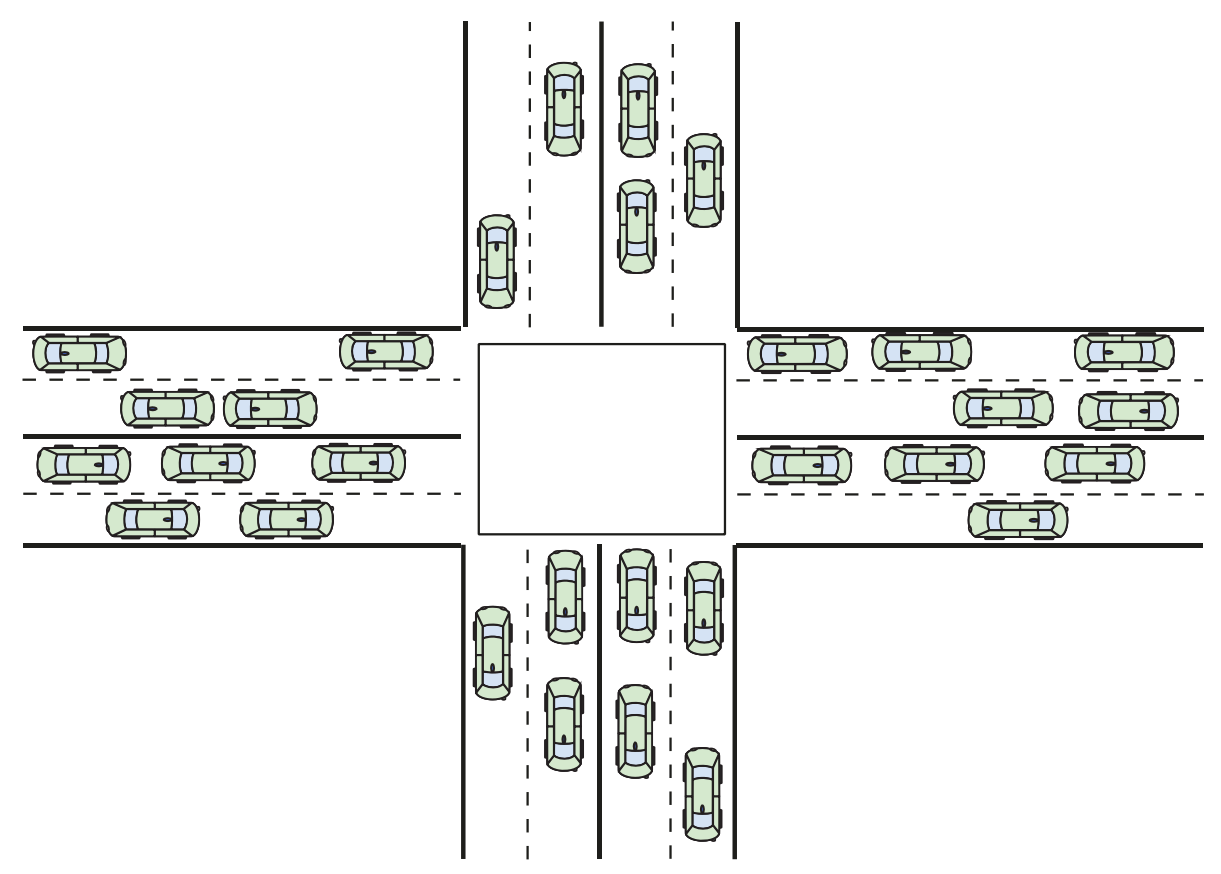

Figure 4: Crossing lanes scenario.

transmit just one packet. We run each simulation for a period of 300 seconds; moreover, for each set of parameters, we repeat the simulation 100 times. Vehicle generation is constant at a rate of 60 vehicles/sec. The number of vehicles in the system is controlled with the minimum distance that must be kept between the new vehicle to be inserted in the lane and the vehicle closest to it. An important issue to consider is that collisions are reported only by the vehicles involved in such collisions; the rest of the vehicles report a hidden terminal since they are only aware that they received a packet. Furthermore, before completing packet reception these vehicles receive one more packet, so they are unable to know if the event was a collision or a hidden terminal. Therefore, in this work we consider these events as a hidden terminal.

3.5. System Parameters. To execute our simulations and evaluate the analytical model of Campolo et al., as well as find the utilization boundaries of the control channel (as a function of the bandwidth) considering the traffic load in the network, we use the discrete event simulator NS3 [22]. This simulator is very well suited for this type of simulations; moreover, it provides the necessary tools to study the performance of IEEE 802.11p since it accounts with the appropriate modules for our analysis. Besides, we added a module to the simulator developed by Arbabi and Weigle [23], which implements the intelligent driver model (IDM) [24]. Furthermore, such a module implements also a lane change model, which is additional to the IDM functionality.

We carry out our simulations considering all the parameters for communications recommended by the IEEE 802.11p standard. Regarding vehicle mobility for both scenarios, we consider the parameters indicated in Table 1.
TABle 1: Parameters considered during our simulations.

\begin{tabular}{lcc}
\hline Parameter & Crossing lanes scenario & Two-way scenario \\
\hline Minimum speed & $8 \mathrm{~m} / \mathrm{s}$ & $8 \mathrm{~m} / \mathrm{s}$ \\
Maximum speed & $13 \mathrm{~m} / \mathrm{s}$ & $25 \mathrm{~m} / \mathrm{s}$ \\
Number of vehicles & $25,50,90,150$ & $25,50,90,150$ \\
\hline
\end{tabular}

Moreover, to obtain meaningful results, we also vary the contention window $(\mathrm{CW})$ with values $\mathrm{CW}=16$ and $\mathrm{CW}=128$ as well as the size of the transmitted packet $(L)$ for values of 300,600 , and 1400 bytes.

\section{Results}

According to the simulations we carry out, we can observe that there is a trend on packet loss for WAVE systems. Figure 5 plots the loss percentage for the two-way scenario for the values of CW and $L$ just mentioned. Notice that the larger the contention window, the smaller the loss rate. We can see in Figure 6 for the crossing lanes scenario a similar behavior, although in this case the loss percentage is greater than the one for the two-way scenario. This is because vehicles in the crossing lanes scenario have a shorter separation; moreover, there are more vehicles in the coverage range of the transmission.

As we have seen, Figure 6 shows a reduction on the loss percentage when increasing the contention window up to $\mathrm{CW}=128$. This is because the wireless devices can choose a greater backoff value; thus, collisions and hidden terminals are reduced at the price of increasing the loss percentage due to channel switching. On the other hand, notice that the packet size also induces a variation on the number 


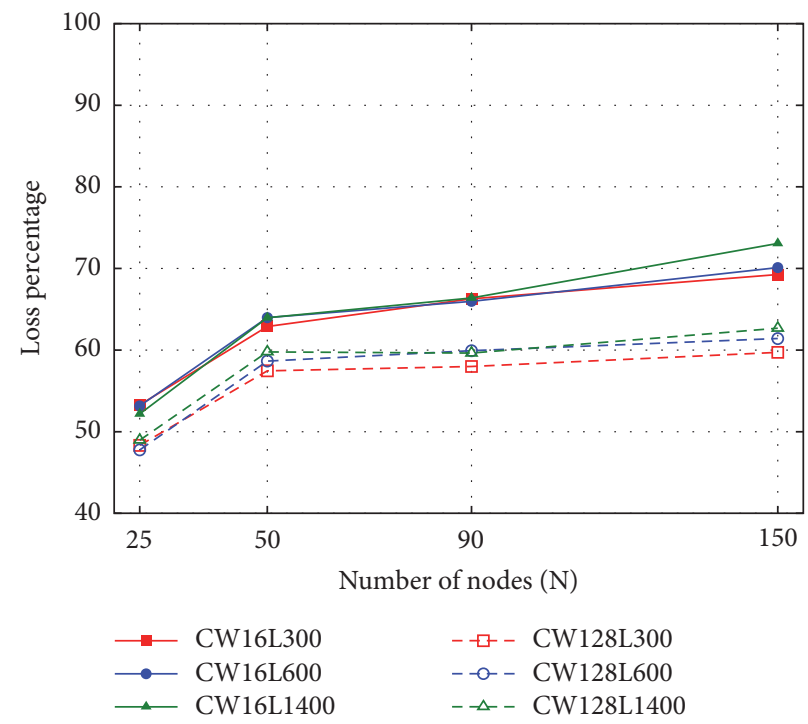

FIGURE 5: Loss percentage versus the number of nodes in the system for the two-way scenario.

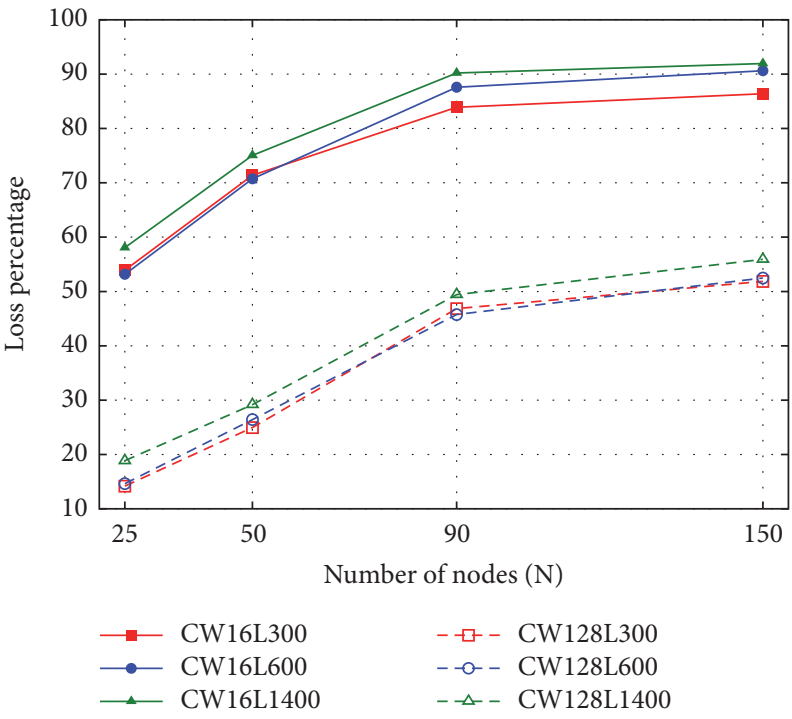

FIGURE 6: Loss percentage versus the number of nodes for the crossing lanes scenario.

of lost packets. This is explained by the fact that as there are more bytes to transmit, it will take longer to complete the transmission, which can cause losses due to channel switching or hidden terminal. The longer the transmission time, the more likely that another vehicle not detecting the medium as busy transmits. This will cause a hidden terminal event or an expiration of the transmit timer; thus, the packet will be lost because of channel switching.

Regarding the crossing lanes scenario, the loss percentage increases. Figure 7 shows the contribution on loss percentage of every type of loss for a contention window $\mathrm{CW}=16$ and packet size $L=300$ bytes. Notice that most of the losses are due to the hidden terminal phenomenon, which occurs when two vehicles located out of the transmission range send their packets, interfering between them at the nodes close to both transmitters. In descending order of contribution to the loss percentage, we can notice losses due to noise, due to collisions, and at the end with a very small contribution we find losses due to the expiration of the control channel.

From these results, it is now clear why the loss rate is greater for the crossing lanes scenario than for the two-way scenario. In the latter, separation between vehicles is greater causing a reduction in the number of collisions and the number of vehicles in a hidden terminal event.

Next, we increase the contention window to a value CW $=128$ and keep the packet size equal to $L=300$ bytes. Figure 8 shows how this configuration reduces the loss rate due to the number of collisions and hidden terminal; also, the loss percentage due to channel switching increases. Although if there is an increase on losses due to channel switching, there is a greater benefit since losses due to 


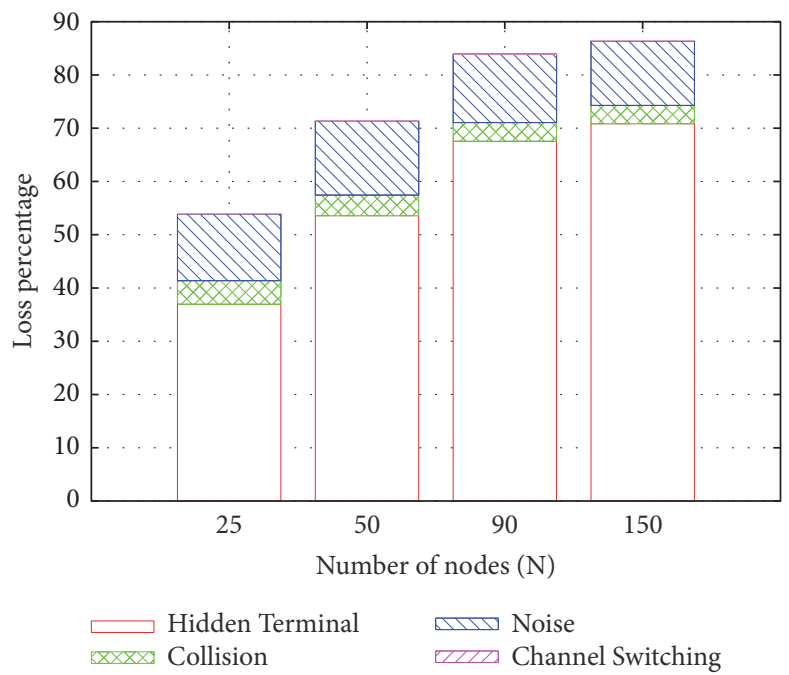

FIGURE 7: Contribution on loss percentage for every type of loss in the system, with CW $=16$ and $L=300$ bytes.

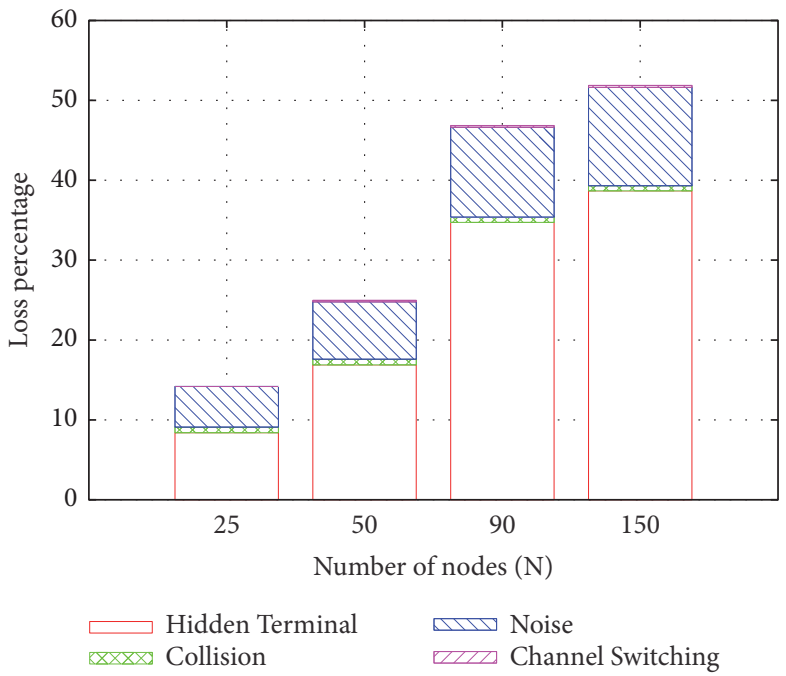

FIGURE 8: Contribution on loss percentage for every type of loss in the system, with CW $=128$ and $L=300$ bytes.

hidden terminal and due to collisions are greatly reduced, compared with the increase on the loss rate due to channel switching.

Up to this point, our results show a great similarity with the findings of Campolo et al. The loss percentage obtained with the crossing lanes scenario in Figure 6 shows a behavior like the one we expect from Figure 2. In both cases, we can infer that when the number of vehicles increases or the packet size increases as well, the loss rate will correspondingly increase. Also, when the contention window grows, the loss percentage will decrease. Despite the similarity in behavior, the expected loss rate with the model of Campolo does not correspond with the loss percentage we find in this work. Especially, losses due to channel switching do not correspond since they do not grow so quickly as expected from model proposed by Campolo. The main reason of the difference in loss rate among both approaches is the strong assumption made in the analytical model. In order to obtain a simplified model, Campolo assumes that nodes are within a reciprocal communication range, meaning that there are no hidden terminals. Also, it is assumed that node mobility does not affect one-hop neighborhood of a node, which is the duration of the control channel, i.e., $50 \mathrm{~ms}$. Therefore, we can observe in the results that these assumptions have undoubtedly an impact on the loss rate of broadcast packets since losses due to hidden terminal have a considerable contribution on the total loss rate.

The most important result we obtain is the fact that when the contention window increases, the loss percentage decreases. To know if this behavior is kept when reaching the maximum contention window recommended in the IEEE 802.11p standard, which is CW $=1024$, we execute an additional simulation campaign for two values of the contention window, $\mathrm{CW}=512$ and $\mathrm{CW}=1024$, with packet sizes $\mathrm{L}=300, \mathrm{~L}=600$, and $\mathrm{L}=1400$. Thus, we plot in Figure 9 the corresponding results. We can easily appreciate that the 


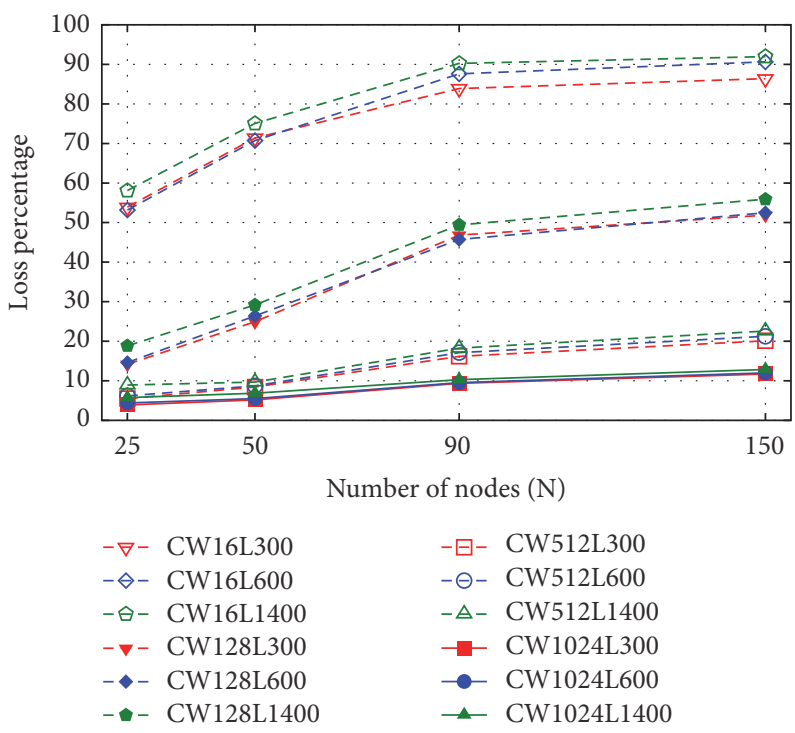

FIGURE 9: Loss percentage versus the number of nodes in the system, with $\mathrm{CW}=[16,128,512,1024]$. Correspondingly, $L=[300,600,1400]$.

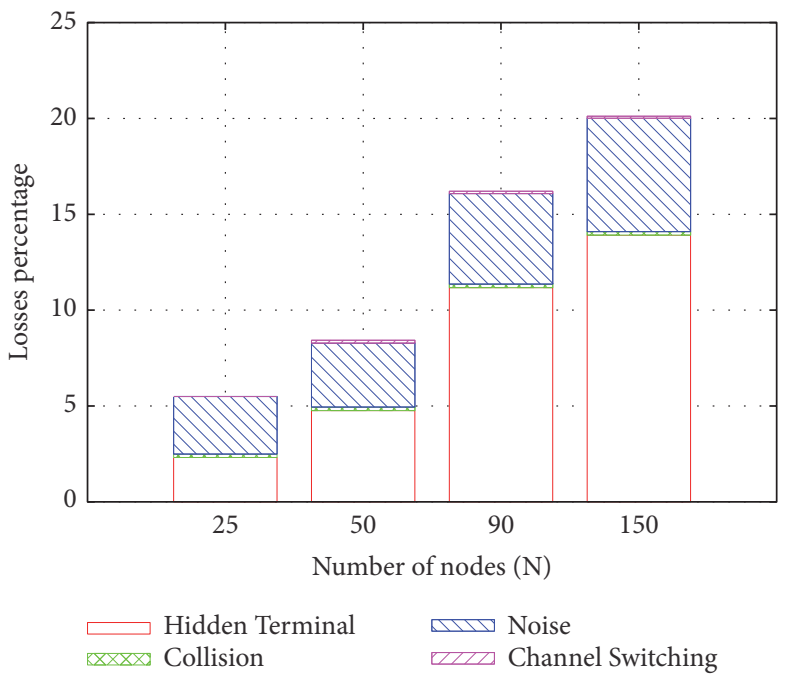

FIgURE 10: Contributions on loss percentage for each type of loss for CW $=512$ and $L=300$.

behavior shown before in Figure 6 is kept. As the contention window grows, the loss percentage reduces.

We can observe the same behavior when analyzing the contribution on loss percentage for each type of loss. Figures 10 and 11 show the reduction on loss percentage due to hidden terminal and collisions when increasing the contention window; losses due to channel switching grow although they increase in a slower way.

Our results suggest a better performance, in terms of the loss percentage, when CW is large. However, there is a tradeoff when CW increases. On one hand, the losses due to channel switching increase. On the other hand, one may expect that with a larger value of CW, the packet would experiment a larger access delay. We define the access delay as the time that a packet remains in the MAC layer, since it waits there until it goes to physical layer. To know the access delay experimented by a packet, simulations are executed for values of $\mathrm{CW}$ in the set $[16,32,64,128,512,1024]$ to get a more detailed information. The results are shown in Figures 12 and 13 , for $L=300$ and $L=1400$, respectively. The results show that the average access delay increases as the number of vehicles increases. This is because the contention window increases with more vehicles trying to transmit. Notice in Figure 12 what happens for 25 vehicles; we see that with a larger value of CW, the average access delay increases. But if we focus on 150 vehicles we can see that when CW increases, the average access delay increases as well, but just until $\mathrm{CW}=64$; then the average access delay decreases. To explain this behavior, we observe in Figure 14 that, for $\mathrm{CW}=16$, the maximum and average access delay show a similar trend. When the number of vehicles increases, the access delay increases as well. But if we focus now on CW $=1024$, the delay growth is lower. This is due to the 50 ms limit for transmission in the control channel. Even if 


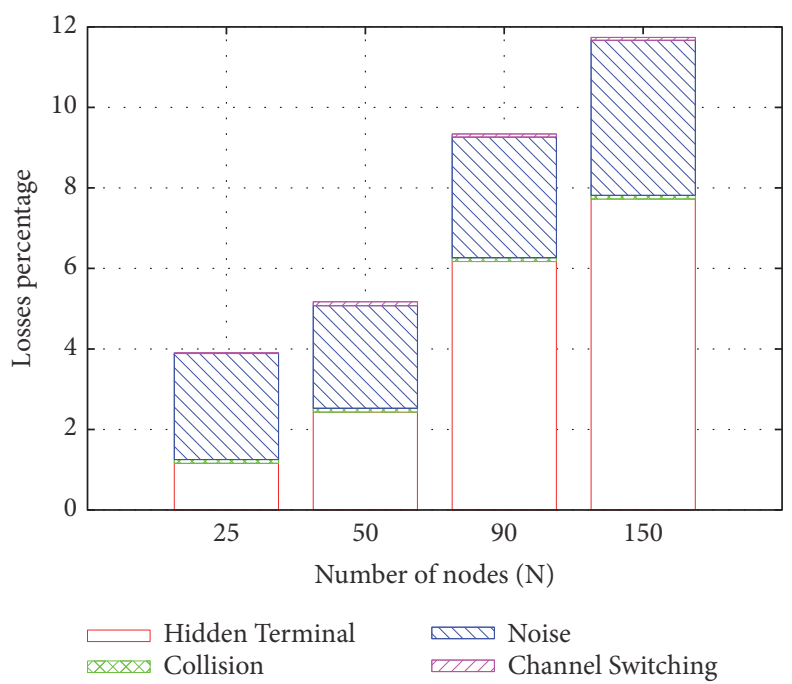

FIGURE 11: Contributions on loss percentage for each type of loss for CW $=1024$ and $L=300$.

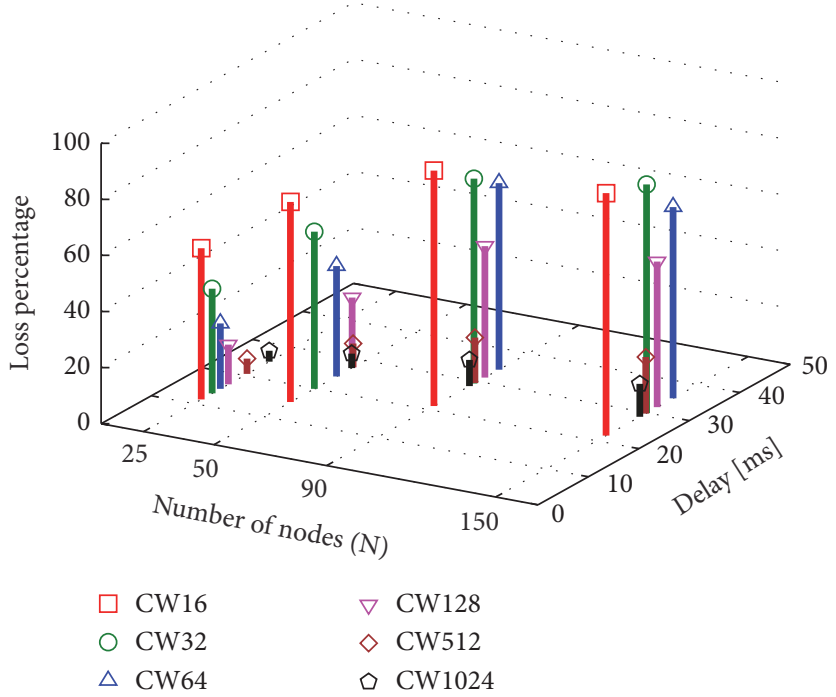

FIGURE 12: Loss percentage and access delay versus number of vehicles for $L=300$.

the contention window size may produce a larger access delay, it cannot exceed $50 \mathrm{~ms}$. Thus, the average access delay is nearly constant. Moreover, with a larger value of CW and a higher number of vehicles, the average access delay experimented by a packet may be less than or equal to a smaller CW.

\section{Conclusions}

In this work, we analyzed the loss process of broadcast packets over the control channel in IEEE 802.11p/WAVE vehicular ad hoc networks. The results obtained allow us to conclude that if the size of the contention window increases, the loss percentage decreases. This is because nodes can choose with higher probability, a different backoff value, which decreases losses due collisions as well as losses due to the hidden terminal phenomenon. Broadcast packet losses are a very important factor for the performance of the control channel in vehicular networks. For most of the messages, an increase in packet delivery time does not have a great impact on network performance; on the contrary, it is very important that messages arrive correctly. Nevertheless, for safety and critical messages the packet delivery time and consequently the loss rate have a great importance. Hence, we found bounds on the contention window size, which can be used according to a specific application. For example, one choice is to set a larger contention window size for control channel and keep a nearly constant access delay. An additional choice is to dynamically adjust the size of the contention window as function of the number of vehicles in the system, the packet size, and the requirements of the message. Furthermore, efficient algorithms for broadcast may reduce the contention window and improve the performance on vehicular networks. 


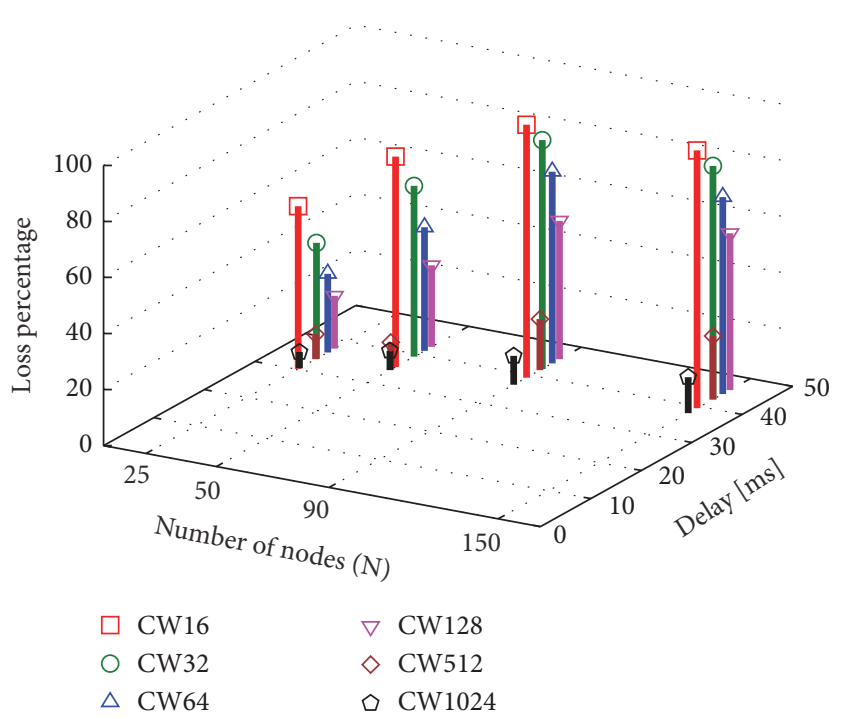

FIGURE 13: Loss percentage and access delay versus number of vehicles for $L=1400$.

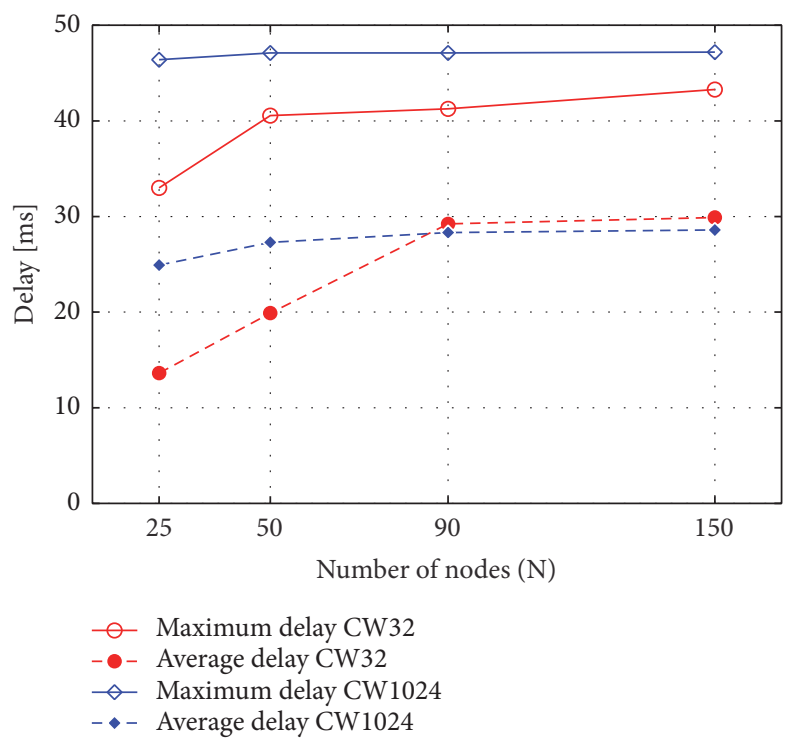

FIGURE 14: Maximum and average access delay for $\mathrm{CW}=32$ and 1024 and $L=300$.

Another important part of this work has been about the analytical model proposed by Campolo et al. Such a model provides a very good approximation to study the impact of the loss process of broadcast packets in the control channel. However, the assumptions made in such a model represent its main drawback since it disregards several important phenomena that occur in vehicular networks. Particularly, to simplify her model Campolo assumes that nodes are within a reciprocal communication range, which means that there are no hidden terminals. This is a strong assumption since in a real scenario one would expect that the hidden terminal phenomenon would occur very frequently on scenarios like the ones we studied in this work. Thus, we analyzed the performance of the system for two general scenarios, one with a two-way vehicular network and another one with a vehicular network with crossing lanes. We carried out extensive simulations campaigns with the NS-3 simulator to find the impact of four types of losses. These types of losses are due to hidden terminal, collisions, noise, and channel switching. In this sense, we explored a wide range of types of losses that cannot be captured with the analytical model.

\section{Data Availability}

Data sharing is not applicable to this article as the resulting statistics were obtained from simulation with the NS-3 simulator.

\section{Conflicts of Interest}

The authors declare that there are no conflicts of interest regarding the publication of this paper. 


\section{Acknowledgments}

This work has been partly supported by the National Council of Science and Technology (CONACyT, Consejo Nacional de Ciencia y Tecnología) of Mexico by providing a Ph.D. scholarship for Salvador Gonzalez.

\section{References}

[1] IEEE Standard for information technology, "IEEE Standard for information technology-local and metropolitan area networks-specific requirements," in Part 11: Wireless LAN Medium Access Control (MAC) and Physical Layer (PHY) Specifications Amendment 6: Wireless Access in Vehicular Environments, Institute of Electrical \&amp; Electronics Engineers (IEEE), 2010.

[2] IEEE Standard for Wireless Access in Vehicular Environments (WAVE)-multi-channel operation, Institute of Electrical \& Electronics Engineers (IEEE), 2011.

[3] C. Campolo, Y. Koucheryavy, A. Molinaro, and A. Vinel, "Characterizing broadcast packet losses in IEEE 802.11p/WAVE vehicular networks," in Proceedings of the 2011 IEEE 22nd International Symposium on Personal, Indoor and Mobile Radio Communications, PIMRC'11, pp. 735-739, Canada, September 2011.

[4] F. A. Teixeira, V. F. e Silva, J. L. Leoni, D. F. Macedo, and J. M. S. Nogueira, "Vehicular networks using the IEEE 802.11p standard: An experimental analysis," Vehicular Communications, vol. 1, no. 2, pp. 91-96, 2014.

[5] R. K. Schmidt, T. Leinmüller, E. Schoch, F. Kargl, and G. Schäfer, "Exploration of adaptive beaconing for efficient intervehicle safety communication," IEEE Network, vol. 24, no. 1, pp. 14-19, 2010.

[6] M. Torrent-Moreno, J. Mittag, P. Santi, and H. Hartenstein, "Vehicle-to-vehicle communication: fair transmit power control for safety-critical information," IEEE Transactions on Vehicular Technology, vol. 58, no. 7, pp. 3684-3703, 2009.

[7] C. Sommer, O. K. Tonguz, and F. Dressler, "Traffic information systems: efficient message dissemination via adaptive beaconing," IEEE Communications Magazine, vol. 49, no. 5, pp. 173-179, 2011.

[8] S. Pallewatta, P. S. Lakmali, S. Wijewardana, P. Ranathunga, T. Samarasinghe, and D. Dias, "802.11p: Insights from the MAC and Physical Layers for a Cooperate Car Following Application," in Intelligent Transport Systems - From Research and Development to the Market Uptake, vol. 222 of Lecture Notes of the Institute for Computer Sciences, Social Informatics and Telecommunications Engineering, pp. 226-236, Springer International Publishing, Cham, 2018.

[9] X. Ma and X. Chen, "Delay and broadcast reception rates of highway safety applications in vehicular ad hoc networks," in Proceedings of the Mobile Networking for Vehicular Environments (MOVE '07), pp. 85-90, Anchorage, Alaska, USA, May 2007.

[10] M. Van Eenennaam, W. K. Wolterink, G. Karagiannis, and G. Heijenk, "Exploring the solution space of beaconing in VANETs," in Proceedings of the 2009 IEEE Vehicular Networking Conference, VNC 2009, pp. 1-8, October 2009.

[11] G. Badawy, J. Misic, T. Todd, and . Dongmei Zhao, "Performance modeling of safety message delivery in vehicular ad hoc networks," in Proceedings of the 2010 IEEE 6th International Conference on Wireless and Mobile Computing, Networking and
Communications (WiMob), pp. 188-195, Niagara Falls, NU, Canada, October 2010.

[12] S. Eichler, "Performance evaluation of the IEEE 802.11 p WAVE communication standard," in Proceedings of the Proceeding of the 66th IEEE Vehicular Technology Conference (VTC '07-Fall), pp. 2199-2203, Baltimore, Md, USA, October 2007.

[13] C. Campolo, A. Vinel, A. Molinaro, and Y. Koucheryavy, "Modeling broadcasting in IEEE 802.11p/WAVE vehicular networks," IEEE Communications Letters, vol. 15, no. 2, pp. 199-201, 2011.

[14] C. Campolo, A. Molinaro, A. Vinel, and Y. Zhang, "Modeling prioritized broadcasting in multichannel vehicular networks," IEEE Transactions on Vehicular Technology, vol. 61, no. 2, pp. 687-701, 2012.

[15] C. Song, "Performance Analysis of the IEEE 802.11p Multichannel MAC Protocol in Vehicular Ad Hoc Networks," Sensors, vol. 17, no. 12, p. 2890, 2017.

[16] H. Jang and W. Feng, "Network Status Detection-Based Dynamic Adaptation of Contention Window in IEEE 802.11p," in Proceedings of the 2010 IEEE 71st Vehicular Technology Conference, pp. 1-5, Taipei, Taiwan, May 2010.

[17] C. Chrysostomou, C. Djouvas, and L. Lambrinos, "Dynamically adjusting the min-max contention window for providing quality of service in vehicular networks," in Proceedings of the 11th Annual Mediterranean Ad Hoc Networking Workshop, Med-HocNet 2012, pp. 16-23, Cyprus, June 2012.

[18] Y. Wang, A. Ahmed, B. Krishnamachari, and K. Psounis, "IEEE 802.11p performance evaluation and protocol enhancement," in Proceedings of the IEEE International Conference on Vehicular Electronics and Safety (ICVES '08), pp. 317-322, IEEE, Columbus, Ohio, USA, September 2008.

[19] D. Garg, R. S. Bali, and A. Kaur, "Performance Evaluation of Data Delivery Mechanism For Cognitive Radio Vehicular and Vehicular Ad-Hoc Networks," Procedia Computer Science, vol. 57, pp. 596-605, 2015.

[20] N. Taherkhani and S. Pierre, "Improving dynamic and distributed congestion control in vehicular ad hoc networks," Ad Hoc Networks, vol. 33, pp. 112-125, 2015.

[21] C. Tripp-Barba, L. Urquiza-Aguiar, A. Zaldívar-Colado, J. Estrada-Jiménez, J. A. Aguilar-Calderón, and M. Aguilar Igartua, "Comparison of propagation and packet error models in vehicular networks performance," Vehicular Communications, vol. 12, pp. 1-13, 2018.

[22] “Network Simulator 3 (NS-3)," Discrete Event Network Simulator, 2018.

[23] H. Arbabi and M. C. Weigle, "Highway mobility and vehicular ad-hoc networks in ns-3," in Proceedings of the 2010 43rd Winter Simulation Conference, WSC'10, pp. 2991-3003, USA, December 2010.

[24] M. Treiber, A. Hennecke, and D. Helbing, "Congested traffic states in empirical observations and microscopic simulations," Physical Review E: Statistical, Nonlinear, and Soft Matter Physics, vol. 62, no. 2 B, pp. 1805-1824, 2000. 


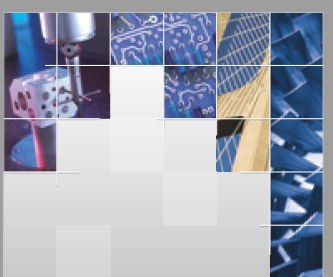

\section{Enfincering}
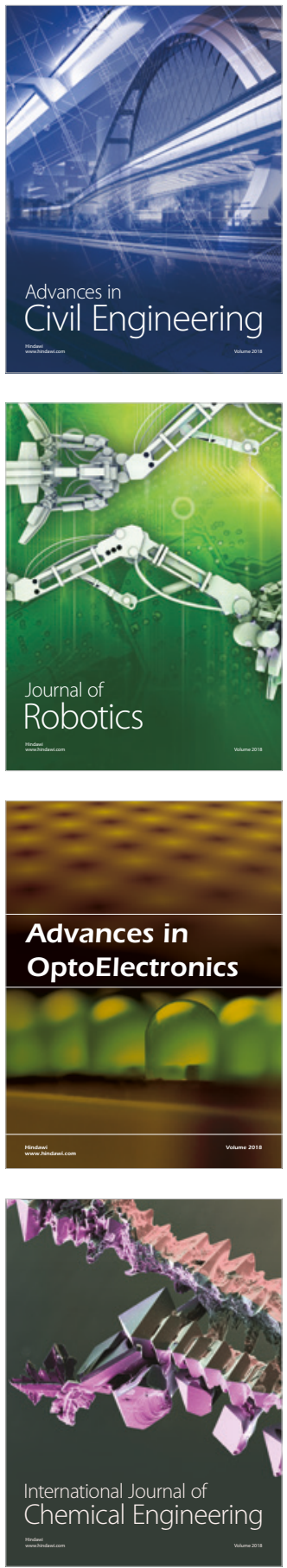

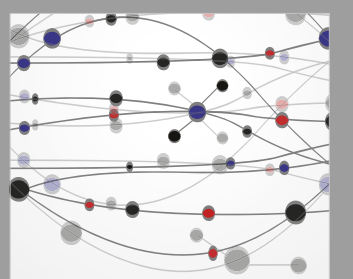

\section{Rotating \\ Machinery}

The Scientific World Journal

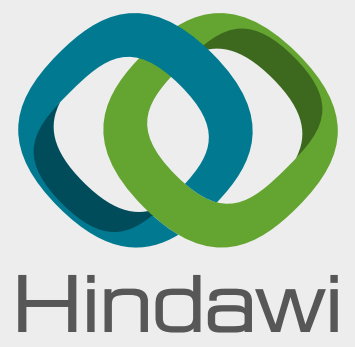

Submit your manuscripts at

www.hindawi.com
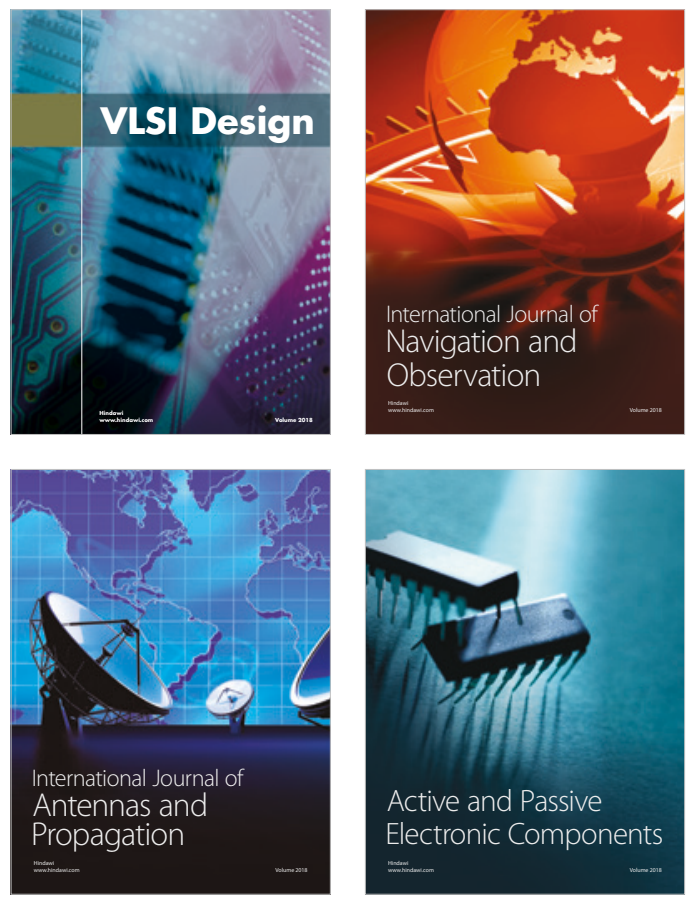
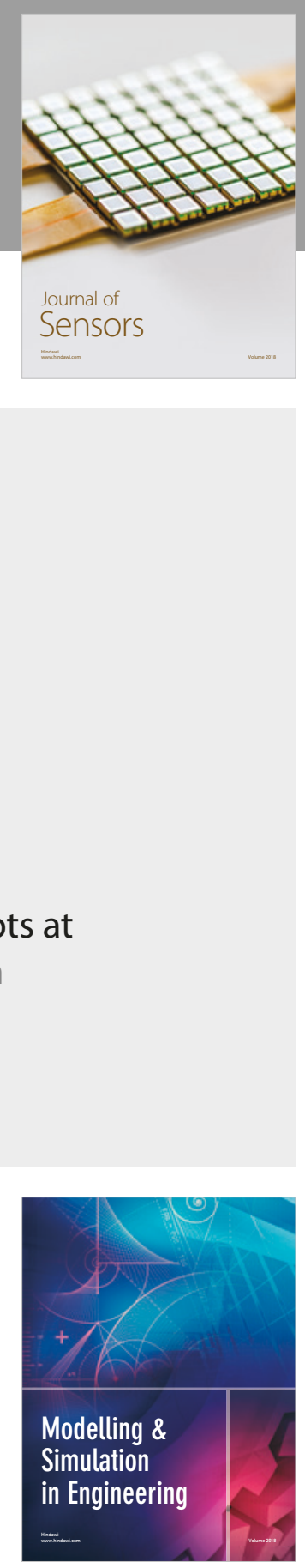

\section{Advances \\ Multimedia}
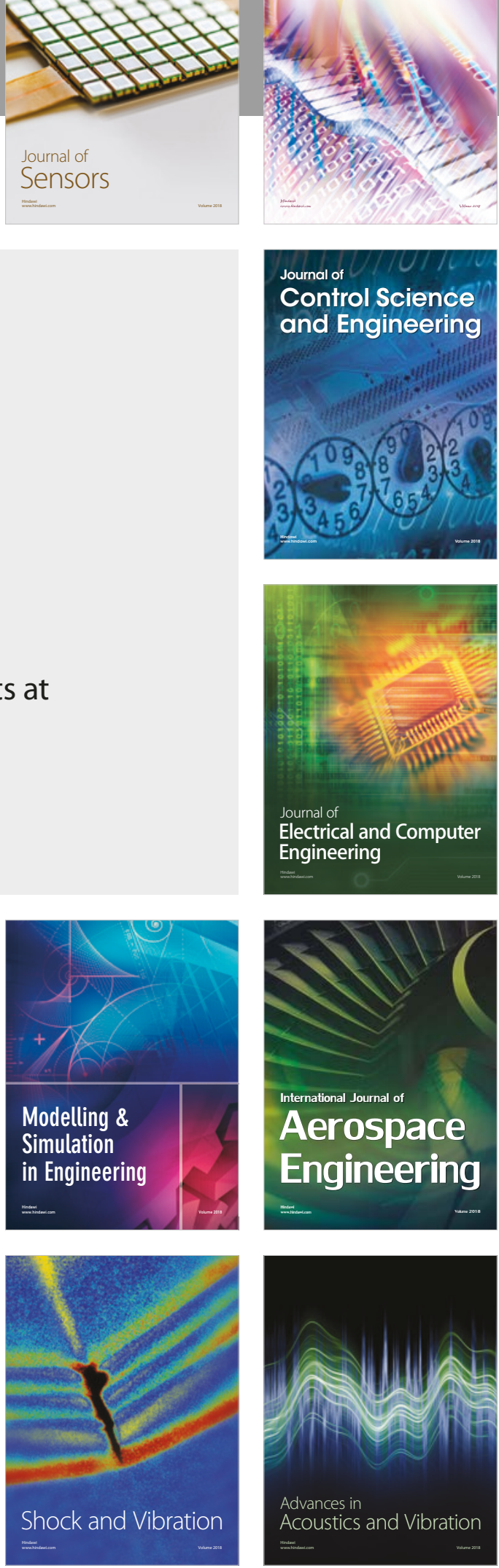